\title{
Toward Extension N-dimensional T-conorms
}

\author{
Eduardo Palmeira, \\ Departamento de Ciências Exatas e Tecnológicas, DCET, UESC \\ 45662-900, Ilhéus, BA \\ E-mail: espalmeira@uesc.br, \\ Benjamín Bedregal \\ Departamento de Informática e Matemática Aplicada, DIMAp, UFRN \\ 59078-970, Natal, RN \\ E-mail: bedregal@dimap.ufrn.br.
}

Resumo: In this paper we apply a method to extend $n$-dimensional lattice-valued $t$-conorms by preserving the largest possible number of properties of these $t$-conorms which are invariants under homomorphisms. Further, we also prove some related results and properties.

Palavras-chave: Extension, $N$-dimensional t-conorm, Lattice

\section{Introdution}

Let $L$ and $K$ be nonempty sets and suppose that $M \subseteq L$. Given a function $f: M \longrightarrow K$, if we want to extend the domain of $f$ to cover the whole $L$, what is the best choice to define $f(x)$ for the elements $x \in L \backslash M$ in order to preserve the largest possible number of properties of $f$ ? This a very complex problem and a answer for this question is not so simple.

Palmeira et al. [8] provided a method to extend t-norms, t-conorms and fuzzy negations using a special mapping (namely e-operator). Also in this work, we apply this extension method for n-dimensional t-norms. As a natural consequence of our researches we discuss here about the extension of n-dimensional t-conorms.

We begin in Section 2 recalling some definitions and results related to lattices, lattice homomorphisms, retractions, sublattices and fuzzy negations. Also in this section, we present the notion of $(r, s)$-sublattices and describe the extension method via e-operators. Sections 3 is devoted to discuss about n-dimensional t-conorms on $L_{n}(L)$ and it extension.

\section{Preliminaries}

Let $L$ be a nonempty set. If $\wedge_{L}$ and $\vee_{L}$ are two binary operations on $L$, then $\left\langle L, \wedge_{L}, \vee_{L}\right\rangle$ is a lattice provided that for each $x, y, z \in L$, the following properties hold:

1. $x \wedge_{L} y=y \wedge_{L} x$ and $x \vee_{L} y=y \vee_{L} x$

2. $\left(x \wedge_{L} y\right) \wedge_{L} z=x \wedge_{L}\left(y \wedge_{L} z\right)$ and $\left(x \vee_{L} y\right) \vee_{L} z=x \vee_{L}\left(y \wedge_{L} z\right)$

3. $x \wedge_{L}\left(x \vee_{L} y\right)=x$ and $x \vee_{L}\left(x \wedge_{L} y\right)=x$.

If in $\left\langle L, \wedge_{L}, \vee_{L}\right\rangle$ there are elements 0 and 1 such that, for all $x \in L, x \wedge_{L} 1=x$ and $x \vee_{L} 0=x$, then $\left\langle L, \wedge_{L}, \vee_{L}, 0,1\right\rangle$ is called a bounded lattice.

Definition 2.1. A homomorphism $r$ of a lattice $L$ onto a lattice $M$ is said to be a retraction if there exists a homomorphism $s$ of $M$ into $L$ which satisfies $r \circ s=i d_{M}$. A lattice $M$ is called a 
retract of a lattice $L$ if there is a retraction $r$, of $L$ onto $M$, and $s$ is then called a pseudo-inverse of $r$.

Definition 2.2. Let $L$ and $M$ be arbitrary bounded lattices. We say that $M$ is a $(r, s)$-sublattice of $L$ if $M$ is a retract of $L$ (i.e. $M$ is a sublattice of $L$ up to isomorphisms). In other words, $M$ is a $(r, s)$-sublattice of $L$ if there is a retraction $r$ of $L$ onto $M$ with pseudo-inverse $s: M \rightarrow L$.

Definition 2.3. Every retraction $r: L \longrightarrow M$ (with pseudo-inverse $s$ ) which satisfies sor $\leqslant i d_{L}{ }^{1}$ $\left(i d_{L} \leqslant s \circ r\right)$ is called a lower (an upper) retraction. In this case, $M$ is a lower (an upper) retract of $L$.

Definition 2.4. Let $M$ be a $\left(r_{1}, s\right)$-sublattice of $L$. If $r_{1}$ is a lower retraction and there is an upper retraction $r_{2}: L \longrightarrow M$ such that its pseudo-inverse is also $s$, then $M$ is called a full $\left(r_{1}, r_{2}, s\right)$-sublattice of $L$. Notation: $M \unlhd L$ with respect to $\left(r_{1}, r_{2}, s\right)$.

Definition 2.5. Let $L$ be a bounded lattice. A binary operation $S: L \times L \longrightarrow L$ is a $t$-conorm if, for all $x, y, z \in L$, it satisfies:

1. $S(x, y)=S(y, x)$ (commutativity);

2. $S(x, S(y, z))=S(S(x, y), z)$ (associativity);

3. If $x \leqslant_{L} y$ then $S(x, z) \leqslant_{L} S(y, z), \forall z \in L$ (monotonicity);

4. $S(x, 0)=x$ (boundary condition);

\subsection{Extension Method via e-operators}

To solve the problem of extending fuzzy logic connectives we have developed in $[6,7,8]$ a special operators which plays a fundamental hole in our extension method. In which follows we define this operator and present some relevant properties of it.

Definition 2.6. Let $M \unlhd L$ with respect to $\left(r_{1}, r_{2}, s\right)$. A mapping $\odot: M \times M \longrightarrow L$ is called an e-operator on $M$ if it is isotonic and satisfies, for each $a, b \in M$ and for each $x \in L$, the following conditions:

$$
\begin{gathered}
r_{1}(a \odot b)=a \wedge_{M} b \text { and } r_{2}(a \odot b)=a \vee_{M} b \\
r_{1}(x) \odot r_{2}(x)=x
\end{gathered}
$$

In other words, if $M \unlhd L$ with respect to $\left(r_{1}, r_{2}, s\right)$ (by Definition 2.4, there are two retractions $r_{1}, r_{2}: L \longrightarrow M$ with the same pseudo-inverse $s: M \longrightarrow L$ such that $\left.s \circ r_{1} \leqslant i d_{L} \leqslant s \circ r_{2}\right)$ the $e$-operator $\odot$ describes an isotonic way to relate retractions $r_{1}$ and $r_{2}$ with the meet and join operators of $M$, respectively, by (1).

Lemma 2.1. Consider $M \unlhd L$ with respect to $\left(r_{1}, r_{2}, s\right)$ and let $\odot$ be an e-operator on $M$. Then, for all $a, b \in M$ and $x, y \in L$, the following properties hold:

1. $a \leqslant_{M} b$ if and only if $r_{1}(a \odot b)=a$ and $r_{2}(a \odot b)=b$;

2. For every $a \in M$ we have $s(a)=a \odot a$;

3.

$$
r_{1}(x) \leqslant_{M} r_{1}(y) \text { and } r_{2}(x) \leqslant_{M} r_{2}(y) \text { iff } x \leqslant_{L} y ;
$$

\footnotetext{
${ }^{1}$ If $f$ and $g$ are functions on a lattice $L$ it is said that $f \leqslant g$ if and only if $f(x) \leqslant_{L} g(x)$ for all $x \in L$.
} 
4. $r_{1}(x)=r_{1}(y)$ and $r_{2}(x)=r_{2}(y)$ if and only if $x=y$;

5. $\odot$ is commutative.

Proposition 2.1. Let $M \unlhd L$ with respect to $\left(r_{1}, r_{2}, s\right)$ and $\odot$ an e-operator on $M$. Thus, if $S$ is a t-conorm on $M$ then

$$
S_{\odot}^{E}(x, y)=S\left(r_{1}(x), r_{1}(y)\right) \odot S\left(r_{2}(x), r_{2}(y)\right)
$$

is a t-conorm on $L$.

Theorem 2.1. Let $M \unlhd L$ with respect to $\left(r_{1}, r_{2}, s\right)$ and $\odot$ be an e-operator on $M$. Thus, given a $t$-norm $T$ on $M$, the function $T_{\odot}^{E}: L^{2} \longrightarrow L$ defined by

$$
T_{\odot}^{E}(x, y)=T\left(r_{1}(x), r_{1}(y)\right) \odot T\left(r_{2}(x), r_{2}(y)\right)
$$

is a t-norm on $L$.

\section{On n-dimensional T-conorms and its Extension}

The $\mathrm{n}$-dimensional fuzzy set theory has been studied as a way to generalize the fuzzy set theory valued to the simplex $L_{n}([0,1])=\left\{x=\left(x_{1}, x_{2}, \ldots, x_{n}\right) \in[0,1]^{n} \mid x_{1} \leqslant x_{2} \leqslant \cdots \leqslant x_{n}\right\}$ for a fixed $n \in \mathbb{N}-\{0\}$ (see [10]). We think about the fuzzy operators (t-norms, t-conorms and fuzzy negations) on $L_{n}([0,1])$. For $n=2$, a good formalization about interval-valued fuzzy logic is given by Deschrijver and partners in $[3,4,5]$. Recent studies for arbitrary $n$ have been done by Bedregal et al. in [2] where a formalization of $\mathrm{n}$-dimensional aggregation functions, particulary t-norms, fuzzy negations and automorphisms on $L_{n}([0,1])$ is carried out.

Based on this framework, an interesting issue is the generalization of lattice-valued aggregation functions to higher dimension using a bounded lattice $L$ instead of $[0,1]$ on the definition of $L_{n}([0,1])$.

One can naturally define a lattice version of the set $L_{n}([0,1])$, namely

$$
L_{n}(L)=\left\{\mathbf{x}=\left(x_{1}, x_{2}, \ldots, x_{n}\right) \in L^{n} \mid x_{1} \leqslant L x_{2} \leqslant L \cdots \leqslant{ }_{L} x_{n}\right\}
$$

where $L$ is a bounded lattice.

For each $\mathbf{x}, \mathbf{y} \in L_{n}(L)$ we define by

$$
\mathbf{x} \wedge \mathbf{y}=\left(x_{1} \wedge_{L} y_{1}, x_{2} \wedge_{L} y_{2}, \ldots, x_{n} \wedge_{L} y_{n}\right)
$$

and

$$
\mathbf{x} \vee \mathbf{y}=\left(x_{1} \vee_{L} y_{1}, x_{2} \vee_{L} y_{2}, \ldots, x_{n} \vee_{L} y_{n}\right)
$$

the meet and join operations on $L_{n}(L)$, respectively.

Denote $/ x /=(x, x, \ldots, x)$ for each $x \in L$. Thus, $/ 0_{L} /$ and $/ 1_{L} /$ are a bottom and a top element of $L_{n}(L)$. As an easy exercise one can prove that $\left\langle L_{n}(L), \wedge, \vee, / 0_{L} /, / 1_{L} /\right\rangle$ is a bounded lattice.

A partial order on $L_{n}(L)$ is given by

$$
\mathbf{x} \leqslant \mathbf{y} \Leftrightarrow x_{i} \leqslant_{L} y_{i} \text { for each } i=1,2, \ldots, n
$$

Proposition 3.1. [2] Let $S_{1}, S_{2}, \ldots, S_{n}:[0,1] \times[0,1] \rightarrow[0,1]$ be $t$-conorms such that $S_{1} \leqslant S_{2} \leqslant$ $\cdots \leqslant S_{n}$. Then

$$
\widetilde{S_{1} \cdots S_{n}}(\mathbf{x}, \mathbf{y})=\left(S_{1}\left(x_{1}, y_{1}\right), \ldots, S_{n}\left(x_{n}, y_{n}\right)\right)
$$

is an $n$-dimensional t-conorm. In case that $S_{1}=S_{2}=\cdots=S_{n}$ we denote $\widetilde{S_{1} \cdots S_{n}}$ by $\mathcal{S}_{S}$. 
A similar result can be easily shown considering a bounded lattice $L$ instead of $[0,1]$ in Proposition 3.1.

In this paper we lead with $\mathcal{S}_{S}$ n-dimensional t-conorm, but every result presented here remains valid for $\widetilde{S_{1} \cdots S_{n}}$.

Corollary 3.1. Let $M \unlhd L$ with respect to $\left(r_{1}, r_{2}, s\right), \odot$ be an e-operator on $M$ and $S$ be a t-conorm on $M$. Then $\mathcal{S}_{S_{\odot}^{E}}: L_{n}(L)^{2} \longrightarrow L_{n}(L)$ given by

$$
\mathcal{S}_{S_{\odot}^{E}}(\mathbf{x}, \mathbf{y})=\left(S_{\odot}^{E}\left(x_{1}, y_{1}\right), S_{\odot}^{E}\left(x_{2}, y_{2}\right), \ldots, S_{\odot}^{E}\left(x_{n}, y_{n}\right)\right)
$$

is a n-dimensional t-conorm on $L_{n}(L)$.

Proof. Straightforward from Theorem 2.1 and Proposition 3.1.

Proposition 3.2. Let $M$ be a $(r, s)$-sublattice of $L$. Then

1. $L_{n}(M)$ is a $(\mathfrak{r}, \mathfrak{s})$-sublattice of $L_{n}(L)$;

2. If $M$ is a lower (upper) $\left(r_{1}, s\right)$-sublattice of $L$ then $L_{n}(M)$ is a lower (upper) $\left(\mathfrak{r}_{1}, \mathfrak{s}\right)$ sublattice of $L_{n}(L)$;

3. If $M \unlhd L$ with respect to $\left(r_{1}, r_{2}, s\right)$ then $L_{n}(M) \unlhd L_{n}(L)$ with respect to $\left(\mathfrak{r}_{1}, \mathfrak{r}_{2}, \mathfrak{s}\right)$ where $\mathfrak{r}_{1}$, $\mathfrak{r}_{2}$ and $\mathfrak{s}$ are suitable homomorphisms defined from $r_{1}, r_{2}$ and $s$ respectively.

Proof. 1. Since $M$ is a $(r, s)$-sublattice of $L$, by Definition 2.2 there is a retraction $r: L \longrightarrow M$ with a pseudo-inverse $s: M \longrightarrow L$ such that $r \circ s=i d_{M}$. Define an n-dimensional function $\mathfrak{r}: L_{n}(L) \longrightarrow L_{n}(M)$ defined for each $\mathbf{x} \in L_{n}(L)$ by

$$
\mathfrak{r}(\mathbf{x})=\left(r\left(x_{1}\right), r\left(x_{2}\right), \ldots, r\left(x_{n}\right)\right)
$$

We claim that $\mathfrak{r}$ is a $n$-dimensional retraction such that its pseudo-inverse is an n-dimensional function $\mathfrak{s}: L_{n}(M) \longrightarrow L_{n}(L)$ given by

$$
\mathfrak{s}(\mathbf{x})=\left(s\left(x_{1}\right), s\left(x_{2}\right), \ldots, s\left(x_{n}\right)\right)
$$

for each $\mathbf{x} \in L_{n}(M)$.

Indeed, it is clear that $\mathfrak{r}$ and $\mathfrak{s}$ are n-dimensional homomorphisms since $r$ and $s$ are. Moreover, for each $\mathbf{x} \in L_{n}(M)$, we have

$$
\begin{aligned}
\mathfrak{r} \circ \mathfrak{s}(\mathbf{x}) & =\mathfrak{r}\left(s\left(x_{1}\right), s\left(x_{2}\right), \ldots, s\left(x_{n}\right)\right) \\
& =\left(r\left(s\left(x_{1}\right)\right), r\left(s\left(x_{2}\right)\right), \ldots, r\left(s\left(x_{n}\right)\right)\right) \\
& =\left(x_{1}, x_{2}, \ldots, x_{n}\right)
\end{aligned}
$$

Thus $\mathfrak{r} \circ \mathfrak{s}=i d_{L_{n}(M)}$ and hence $L_{n}(M)$ is a $(\mathfrak{r}, \mathfrak{s})$-sublattice of $L_{n}(L)$ by Definition 2.2.

2. If $M$ is a lower $\left(r_{1}, s\right)$-sublattice of $L$ then $s \circ r_{1} \leqslant i d_{L}$. We shall prove that $\mathfrak{s} \circ \mathfrak{r}_{1} \leqslant i d_{L_{n}(L)}$. Thus, for each $\mathbf{x} \in L_{n}(L)$ it follows that

$$
\begin{aligned}
\mathfrak{s} \circ \mathfrak{r}_{1}(\mathbf{x}) & =\mathfrak{s}\left(r_{1}\left(x_{1}\right), r_{1}\left(x_{2}\right), \ldots, r_{1}\left(x_{n}\right)\right) \\
& =\left(s\left(r_{1}\left(x_{1}\right)\right), s\left(r_{1}\left(x_{2}\right)\right), \ldots, s\left(r_{1}\left(x_{n}\right)\right)\right) \\
& \leqslant\left(x_{1}, x_{2}, \ldots, x_{n}\right)
\end{aligned}
$$

Analogously, one can prove that $L_{n}(M)$ is an upper $\left(\mathfrak{r}_{2}, \mathfrak{s}\right)$-sublattice of $L_{n}(L)$ assuming that $M$ is an upper $\left(r_{2}, s\right)$-sublattice of $L$. 
3. Suppose that $M \unlhd L$. Thus, there are a lower and an upper retractions $r_{1}$ and $r_{2}$ from $L$ onto $M$ with the same pseudo-inverse $s: M \longrightarrow L$. Therefore, by items 1 . and 2 . it is easy to check that $\mathfrak{r}_{\mathbf{1}}(\mathbf{x})=\left(r_{1}\left(x_{1}\right), r_{1}\left(x_{2}\right), \ldots, r_{1}\left(x_{n}\right)\right)$ and $\mathfrak{r}_{2}(\mathbf{x})=\left(r_{2}\left(x_{1}\right), r_{2}\left(x_{2}\right), \ldots, r_{2}\left(x_{n}\right)\right)$ for each $\mathbf{x} \in L_{n}(L)$ are $\mathrm{n}$-dimensional lower and upper retractions with the same n-dimensional pseudo-inverse $\mathfrak{s}(\mathbf{x})=\left(s\left(x_{1}\right), s\left(x_{2}\right), \ldots, s\left(x_{n}\right)\right)$ for each $\mathbf{x} \in L_{n}(M)$ according to which it can be inferred that $L_{n}(M) \unlhd L_{n}(L)$ with respect to $\left(\mathfrak{r}_{1}, \mathfrak{r}_{2}, \mathfrak{s}\right)$.

Proposition 3.3. Let $M \unlhd L$ with respect to $\left(r_{1}, r_{2}, s\right)$ and let $\odot$ be an e-operator on $M$. The function $\odot^{n}: L_{n}(M) \times L_{n}(M) \longrightarrow L_{n}(L)$ given by

$$
\mathbf{a} \odot{ }^{n} \mathbf{b}=\left(a_{1} \odot b_{1}, a_{2} \odot b_{2}, \ldots, a_{n} \odot b_{n}\right)
$$

for all $\mathbf{a}, \mathbf{b} \in L_{n}(M)$ is an e-operator on $L_{n}(M)$.

Proof. Straightforward from Proposition 3.2 and from the fact that $\odot$ is an $e$-operator on $M$.

Corollary 3.2. Let $M \unlhd L$ with respect to $\left(r_{1}, r_{2}, s\right)$ and $\odot$ be an e-operator on $M$. If $\mathcal{S}$ is a t-conorm on $L_{n}(M)$ then the function $\mathcal{S}_{\odot}^{E}: L_{n}(L) \times L_{n}(L) \longrightarrow L_{n}(L)$ given by

$$
\mathcal{S}_{\odot}^{E}(\mathbf{x}, \mathbf{y})=\mathcal{S}\left(\mathfrak{r}_{1}(\mathbf{x}), \mathfrak{r}_{1}(\mathbf{y})\right) \odot^{n} \mathcal{S}\left(\mathfrak{r}_{2}(\mathbf{x}), \mathfrak{r}_{2}(\mathbf{y})\right)
$$

for all $\mathbf{x}, \mathbf{y} \in L_{n}(L)$, is a t-conorm on $L_{n}(L)$.

Proof. Straightforward from Theorem 2.1 and Propositions 3.2 and 3.3.

Theorem 3.1. Let $M \unlhd L$ with respect to $\left(r_{1}, r_{2}, s\right), \odot$ be an e-operator on $M$ and $S$ be a t-conorm on $M$. Then $\left(\mathcal{S}_{S}\right)_{\odot}^{E}=\mathcal{S}_{S_{\odot}^{E}}$.

Proof. Take $\mathbf{x}, \mathbf{y} \in L_{n}(L)$. Then,

$$
\begin{array}{rlrl}
\left(\mathcal{S}_{S}\right) \stackrel{E}{E}(\mathbf{x}, \mathbf{y})= & \mathcal{S}_{S}\left(\mathfrak{r}_{\mathbf{1}}(\mathbf{x}), \mathfrak{r}_{1}(\mathbf{y})\right) \odot^{n} \mathcal{S}\left(\mathfrak{r}_{2}(\mathbf{x}), \mathfrak{r}_{2}(\mathbf{y})\right) & \text { by }(5) \\
= & \mathcal{S}_{S}\left(\left(r_{1}\left(x_{1}\right), \ldots, r_{1}\left(x_{n}\right)\right),\left(r_{1}\left(y_{1}\right), \ldots, r_{1}\left(y_{n}\right)\right) \odot^{n}\right. & \\
& \mathcal{S}_{S}\left(\left(r_{2}\left(x_{1}\right), \ldots, r_{2}\left(x_{n}\right)\right),\left(r_{2}\left(y_{1}\right), \ldots, r_{2}\left(y_{n}\right)\right)\right. & & \text { by eq. }(8) \\
= & \left(S\left(r_{1}\left(x_{1}\right), r_{1}\left(y_{1}\right)\right), \ldots, S\left(r_{1}\left(x_{n}\right), r_{1}\left(y_{n}\right)\right)\right) \odot^{n} & \\
& \left(S\left(r_{2}\left(x_{1}\right), r_{2}\left(y_{1}\right)\right), \ldots, S\left(r_{2}\left(x_{n}\right), r_{2}\left(y_{n}\right)\right)\right) & & \text { by eq. }(7) \\
= & \left(S\left(r_{1}\left(x_{1}\right), r_{1}\left(y_{1}\right)\right) \odot S\left(r_{2}\left(x_{1}\right), r_{2}\left(y_{1}\right)\right), \ldots,\right. & \\
& S\left(r_{1}\left(x_{n}\right), r_{1}\left(y_{n}\right)\right) \odot S\left(r_{2}\left(x_{n}\right), r_{2}\left(y_{n}\right)\right) & & \text { by eq. }(9) \\
= & \left(S_{\odot}^{E}\left(x_{1}, y_{1}\right), \ldots, S_{\odot}^{E}\left(x_{n}, y_{n}\right)\right) & \text { by eq. }(5) \\
= & \mathcal{S}_{S_{\odot}^{E}(\mathbf{x}, \mathbf{y})} & \text { by eq. }(7)
\end{array}
$$

Let $\mathfrak{C}_{M}$ be the set of all t-conorms $S$ on $M$ (similar to $\mathfrak{C}_{L}, \mathfrak{C}_{L_{n}(M)}$ and $\left.\mathfrak{C}_{L_{n}(L)}\right)$. The theorem above shows that the following diagram is commutative:

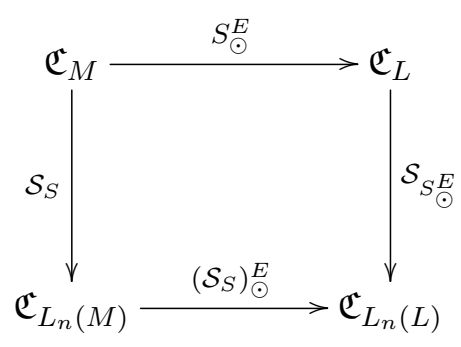


Proposition 3.4. Let $L$ be a bounded lattice. For all $n \in \mathbb{N}-\{0\}$ we have $L_{m}(L) \unlhd L_{n}(L)$ with respect to some $\left(\mathfrak{r}_{1}, \mathfrak{r}_{1}, \mathfrak{s}\right)$ when $n=2 m$. Moreover, the mapping $\odot: L_{m}(L) \times L_{m}(L) \longrightarrow L_{n}(L)$ defined by

$$
\left(x_{1}, \ldots, x_{m}\right) \odot\left(y_{1}, \ldots, y_{m}\right)=\left(x_{1} \wedge_{L} y_{1}, x_{1} \vee_{L} y_{1}, \ldots, x_{m} \wedge_{L} y_{m}, x_{m} \vee_{L} y_{m}\right)
$$

is an e-operator.

Proof. We shall present an n-dimensional lower retraction $\mathfrak{r}_{1}: L_{n}(L) \longrightarrow L_{m}(L)$, an n-dimensional upper retraction $\mathfrak{r}_{2}: L_{n}(L) \longrightarrow L_{m}(L)$ and an n-dimensional pseudo-inverse $\mathfrak{s}: L_{m}(L) \longrightarrow$ $L_{n}(L)$ such that $\mathfrak{s} \circ \mathfrak{r}_{1} \leqslant i d_{L_{n}(L)} \leqslant \mathfrak{s} \circ \mathfrak{r}_{2}$. Let

$$
\mathfrak{r}_{1}\left(x_{1}, \ldots, x_{n}\right)=\left(x_{1}, x_{3}, \ldots, x_{n-1}\right)
$$

and

$$
\mathfrak{r}_{2}\left(x_{1}, \ldots, x_{n}\right)=\left(x_{2}, x_{4}, \ldots, x_{n}\right)
$$

for all $\left(x_{1}, \ldots, x_{n}\right) \in L_{n}(L)$. It is clear that $\mathfrak{r}_{1}$ and $\mathfrak{r}_{2}$ are homomorphisms. Moreover, the function given by $\mathfrak{s}\left(x_{1}, x_{2}, \ldots, x_{m}\right)=\left(x_{1}, x_{1}, x_{2}, x_{2}, \ldots, x_{m}, x_{m}\right)$ is an homomorphism such that

$$
\begin{aligned}
\mathfrak{s} \circ \mathfrak{r}_{1}\left(x_{1}, \ldots, x_{n}\right) & =\mathfrak{s}\left(x_{1}, x_{3}, \ldots, x_{n-1}\right) \\
& =\left(x_{1}, x_{1}, x_{3}, x_{3}, \ldots, x_{n-1}, x_{n-1}\right) \\
& \leqslant\left(x_{1}, x_{2}, x_{3}, x_{4}, \ldots, x_{n}\right)
\end{aligned}
$$

and

$$
\begin{aligned}
\mathfrak{r}_{1} \circ \mathfrak{s}\left(x_{1}, x_{2}, \ldots, x_{m}\right) & =\mathfrak{r}_{1}\left(x_{1}, x_{1}, x_{2}, x_{2}, \ldots, x_{m}, x_{m}\right) \\
& =\left(x_{1}, x_{2}, \ldots, x_{m}\right) \\
& =i d_{L_{m}(L)}\left(x_{1}, x_{2}, \ldots, x_{m}\right)
\end{aligned}
$$

Therefore, $\mathfrak{r}_{1}$ is an $n$-dimensional lower retraction which pseudo-inverse is $\mathfrak{s}$.

Analogously, it can be proved that $\mathfrak{r}_{2}$ is an $n$-dimensional upper retraction with pseudoinverse $\mathfrak{s}$.

On the other hand,

1. clearly $\odot$ is isotonic;

2. $\mathfrak{r}_{1}\left(\left(x_{1}, \ldots, x_{m}\right) \odot\left(y_{1}, \ldots, y_{m}\right)\right)=\mathfrak{r}_{1}\left(x_{1} \wedge_{L} y_{1}, x_{1} \vee_{L} y_{1}, \ldots, x_{m} \wedge_{L} y_{m}, x_{m} \vee_{L} y_{m}\right)=\left(x_{1} \wedge_{L}\right.$ $\left.y_{1}, \ldots, x_{m} \wedge_{L} y_{m}\right)=\left(x_{1}, \ldots, x_{m}\right) \wedge_{L_{m}(L)}\left(y_{1}, \ldots, y_{m}\right)$

3. $\mathfrak{r}_{2}\left(\left(x_{1}, \ldots, x_{m}\right) \odot\left(y_{1}, \ldots, y_{m}\right)\right)=\left(x_{1}, \ldots, x_{m}\right) \vee_{L_{m}(L)}\left(y_{1}, \ldots, y_{m}\right)$; and

4. $\mathfrak{r}_{1}\left(x_{1}, \ldots, x_{n}\right) \odot \mathfrak{r}_{1}\left(x_{1}, \ldots, x_{n}\right)=\left(x_{1}, x_{3}, \ldots, x_{n-1}\right) \odot\left(x_{2}, x_{4}, \ldots, x_{n}\right)=\left(x_{1} \wedge_{L} x_{2}, x_{1} \vee_{L}\right.$ $\left.x_{2}, \ldots, x_{n-1} \wedge_{L} x_{n}, x_{n-1} \vee_{L} x_{n}\right)=\left(x_{1}, x_{2}, \ldots, x_{n}\right)$.

Therefore, $\odot$ is an e-operator.

Notice that there are other possibilities for $m$ and $n$ such that $L_{m}(L) \unlhd L_{n}(L)$. For example, $m=5$ and $n=8$. In this case $\mathfrak{r}_{1}\left(x_{1}, \ldots, x_{8}\right)=\left(x_{1}, x_{3}, x_{5}, x_{7}, x_{8}\right), \mathfrak{r}_{2}\left(x_{1}, \ldots, x_{8}\right)=$ $\left(x_{1}, x_{2}, x_{4}, x_{6}, x_{8}\right)$ and $\mathfrak{s}\left(x_{1}, \ldots, x_{5}\right)=\left(x_{1}, x_{2}, x_{2}, x_{3}, x_{3}, x_{4}, x_{4}, x_{5}\right)$.

Corollary 3.3. Let $\mathcal{S}$ be a t-conorm on $L_{m}(L)$ and $n=2 m$. The function $\mathcal{S}_{\odot}^{E}: L_{n}(L) \times$ $L_{n}(L) \longrightarrow L_{n}(L)$ given by

$$
\mathcal{S}_{\odot}^{E}(\mathbf{x}, \mathbf{y})=\mathcal{S}\left(\mathfrak{r}_{1}(\mathbf{x}), \mathfrak{r}_{1}(\mathbf{y})\right) \odot \mathcal{S}\left(\mathfrak{r}_{2}(\mathbf{x}), \mathfrak{r}_{2}(\mathbf{y})\right)
$$

for all $\mathbf{x}, \mathbf{y} \in L_{n}(L)$, is a t-conorm on $L_{n}(L)$.

Proof. Straightforward from Theorem 2.1 and Proposition 3.4. 


\section{Final Remarks}

The method of extending fuzzy connectives via $e$-operators presented in [8] proved to be efficient to solve the challenge of defining extensions that are able to preserve the largest possible number of properties of fuzzy connectives. This good results remain valid for n-dimensional t-conorms, as we could see in this paper.

For further works, we would like still applying this extension method for other fuzzy operators in order to test its efficiency in preserving the main properties of these operators.

\section{References}

[1] B. C. Bedregal. On Interval Fuzzy Negations. Fuzzy Sets and Systems, 161:2290-2313, 2010.

[2] B. C. Bedregal, G. Beliakov, H. Bustince, T. Calvo, R. Mesiar and D. Paternain. A Class of Fuzzy Multisets with a Fixed Number of Memberships. Information Science, 189:1-17, 2012.

[3] G. Deschrijver. The Archimedean property for t-norms in interval-valued fuzzy set theory. Fuzzy Sets and Systems, 157(17): 2311-2327, 2006.

[4] G. Deschrijver. A representation of t-norms in interval-valued L-fuzzy set theory. Fuzzy Sets and Systems, 159(13): 1597-1618, 2008.

[5] G. Deschrijver. Triangular norms which are meet-morphisms in interval-valued fuzzy set theory. Fuzzy Sets and Systems, 181(1): 88-101, 2011.

[6] E. S. Palmeira and B. C. Bedregal. Extension of Fuzzy Logic Operators Defined on Bounded Lattices via Retractions. Computer $\&$ Mathematics with Applications, 63: 1026-1038, 2012.

[7] E. S. Palmeira, B. C. Bedregal, J. Fernandez and A. Jurio. On the Extension of Latticevalued Implications via Retractions. Fuzzy Sets and Systems, 2013 (in press).

[8] E. S. Palmeira, B. C. Bedregal, R. Mesiar and J. Fernandez. A New Way to Extend T-norms, T-conorms and Negations. Fuzzy Sets and Systems, 2013 (in press).

[9] Saminger-Platz, S., Klement, E.P., Mesiar, R.:On Extension of Triangular Norms On Bounded Lattices. John Wiley, New York, 2004.

[10] Y. Shang, X. Yuan and E. S. Lee. The n-Dimensional Fuzzy Sets and Zadeh Fuzzy Sets Based on the Finite Valued Fuzzy Sets. Computers 85 Mathematics with Applications, 60: 442-463, 2010. 